\title{
Patterned Absorption Layers for Pyroelectric Detectors with Higher Sensitivity
}

\author{
Yvonne Querner, Volkmar Norkus, Gerald Gerlach \\ Technische Universität Dresden, Institut für Festkörperelektronik, 01062 Dresden
}

\begin{abstract}
A basic requirement for achieving a high sensitivity of pyroelectric detectors is the effective conversion of incident infrared radiation into heat. In many applications absorption layers of different metals like silver, gold, nickel chrome, or platinum are used to increase the sensitivity of the pyroelectric sensor. Also, polymer coatings with special filler materials like carbon black are widely used for increasing the absorption properties in the infrared range.

These methods have several disadvantages e.g. some of the layers are not mechanically stable, are not resistant to solvents and are not long-term stable, others require a time-consuming preparation.

This study investigates the ability to pattern polymer layers 3-dimensionally on the one hand in order to improve the optical properties of the absorption layer and on the other hand to avoid the above mentioned problems.
\end{abstract}

Keywords: $\quad$ Pyroelectric detector, absorption layers, patterned layers, high sensitivity

\section{Introduction}

Pyroelectric detectors are widely used in many applications. Main fields of application are for example the safety sector, spectroscopy, pyrometry and gas analysis. For all applications a high sensitivity of the detector is of great interest.

Since only the absorbed portion of the incident radiation is available for the signal generation in the sensor the absorption coefficient of the sensitive element influences the characteristics of the pyroelectric sensor in a great extent.

In general, the responsivity of thermal detectors is directly proportional to the absorption coefficient of the sensitive element $(R \sim \alpha)$ [1]. There are many possibilities to increase the absorption. For example, in many applications absorption layers of different porous metals like silver, gold, nickel chrome, or platinum are used. Also, polymer coatings with special filler materials like carbon black are widely used for increasing the absorption properties in the infrared range [2]. But many of the layers are not mechanically stable, are not resistant to solvents and are not long-term stable.

The production of polymer layers with additives is sometimes also very time-consuming. To produce such layers first a suspension of the polymer and the filler material has to be prepared. For a better homogeneity it has to be treated in an ultrasonic bath for about 30 minutes. Due to the better absorption properties of the filler material, compared to the polymer matrix in the exposure range the exposure time for the polymer can be very long, or the development results are not satisfying so that a second exposure and development step is necessary.

\section{Micro-patterned absorption layers}

In order to avoid these disadvantages this study investigates the possibilities to find an alternative method for increasing the absorption properties of pyroelectric detectors by patterning the attached polymer layer on the sensitive element. The approach is aimed to reduce reflection of the absorption layer due to its particular pattern and hence to increase sensitivity. This should be achieved by a pure polymer film without any filling material or additive. 


\section{Absorption Layer Deposition and Patterning}

The structures in the polymer layer should refract the incident radiation in such a way that the reflected part could be absorbed again in the layer. They should work similar to moth eyes that have a continuous refractive index gradient and hence decrease the reflection.

Reduced reflection of the coating means higher absorption and hence the responsivity will be increased.

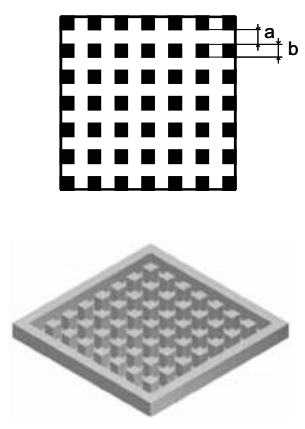

Figure 1: a) columns
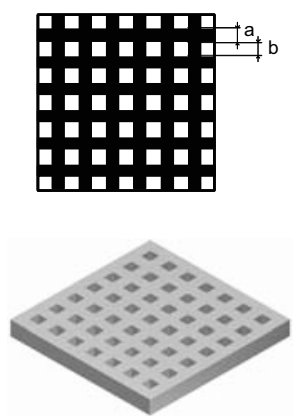

b) holes
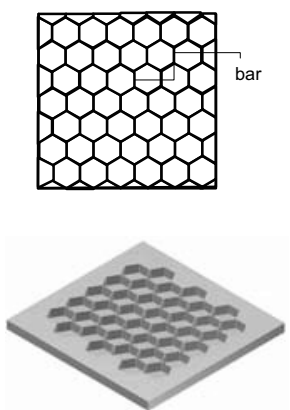

c) honeycomb pattern

The inserted structures form columns (fig. 1a), holes (fig. 1b) and honeycomb (fig. 1c) pattern. The exact dimensions of the structures are shown in table 1.

Table 1: Dimensions of investigated patterned absorption layers (cp. Fig.1)

\begin{tabular}{|l|c|c|}
\hline Pattern & structure width a & distance $\mathrm{b}$ between structures \\
\hline Holes & $6,8 \mu \mathrm{m}$ & $8,2 \mu \mathrm{m}$ \\
\hline Columns & $8,2 \mu \mathrm{m}$ & $6,8 \mu \mathrm{m}$ \\
\hline Honeycomb & $10 \mu \mathrm{m}$ (wall thickness) & $200 \mu \mathrm{m}$ (diameter) \\
\hline
\end{tabular}

The polymer layers are spin-coated on the sensitive element of the pyroelectric detector. The polymer is a novolak from Allresist (X-AR-P 3250/5). They are about $5 \mu \mathrm{m}$ thick. Afterwards they are patterned by a lithography process. The exposure time and the development time take both about 60 s. Three different patterns were investigated. They are shown in figure 1.

\section{Experimental Results}

In order to characterise the influence of the structures the polymer layer were spin-coated and patterned on $\mathrm{CaF}_{2}$-carriers. The advantage of $\mathrm{CaF}_{2}$-carriers is the transmission of nearly $100 \%$ in the interesting infrared range between 2 and $14 \mu \mathrm{m}$ in order to determine the optical properties of the layers directly. Transmission $\mathrm{T}$ and reflectance $\rho$ of the structured layers were measured by FTIR. The absorbance is then given by:

$$
\alpha=1-\mathrm{T}-\rho
$$

Figures $2 a-2 c$ present the absorbance of the patterned investigated polymer layers.

The results show an improvement of the optical properties of patterned polymer layers in contrast to such ones without any structures. The honeycomb structure has proved to be not suitable. The amount of polymer is not sufficient to absorb large enough amounts of the incident radiation. The absorbance could not be improved. The column structure shows only in the range of $2 \ldots 4 \mu \mathrm{m}$ an improvement, but not as significant as the hole structure which showed the best absorption (Fig. 2a). Broadband IR absorbance of $70 . . .80 \%$ of these polymer layers was achieved. 


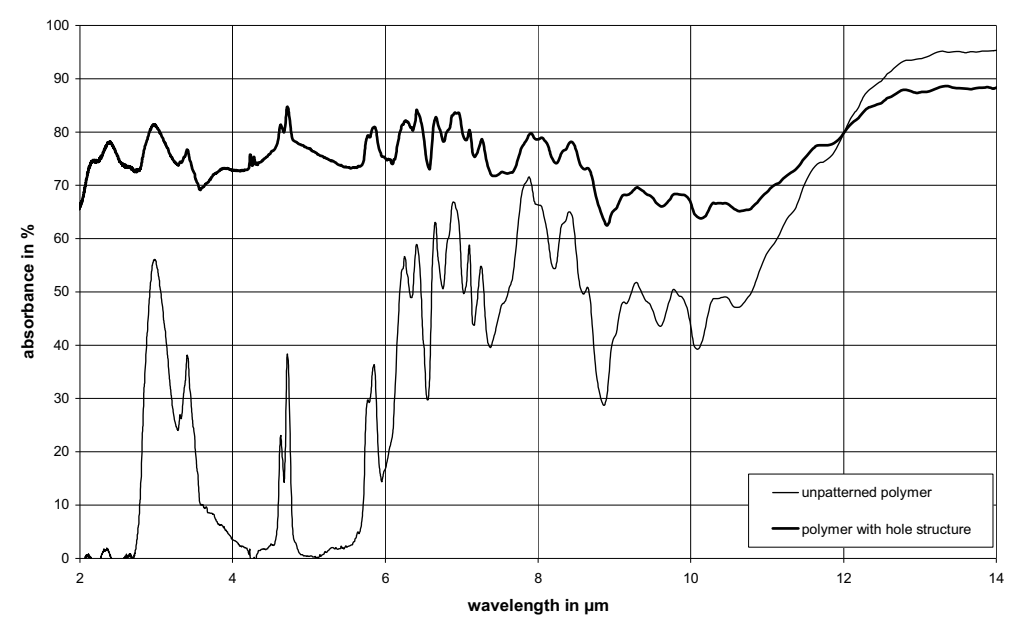

a)

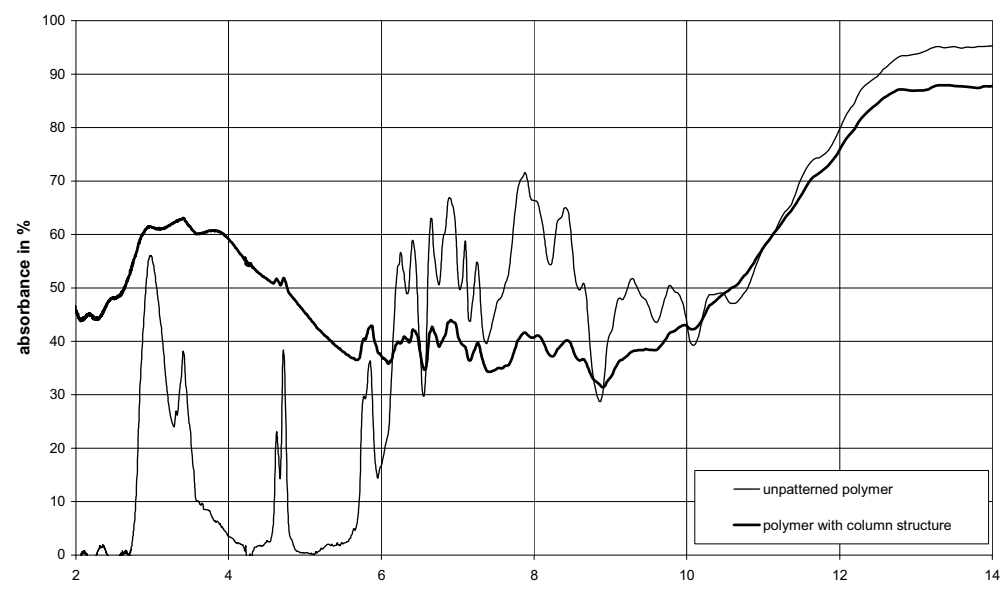

b)
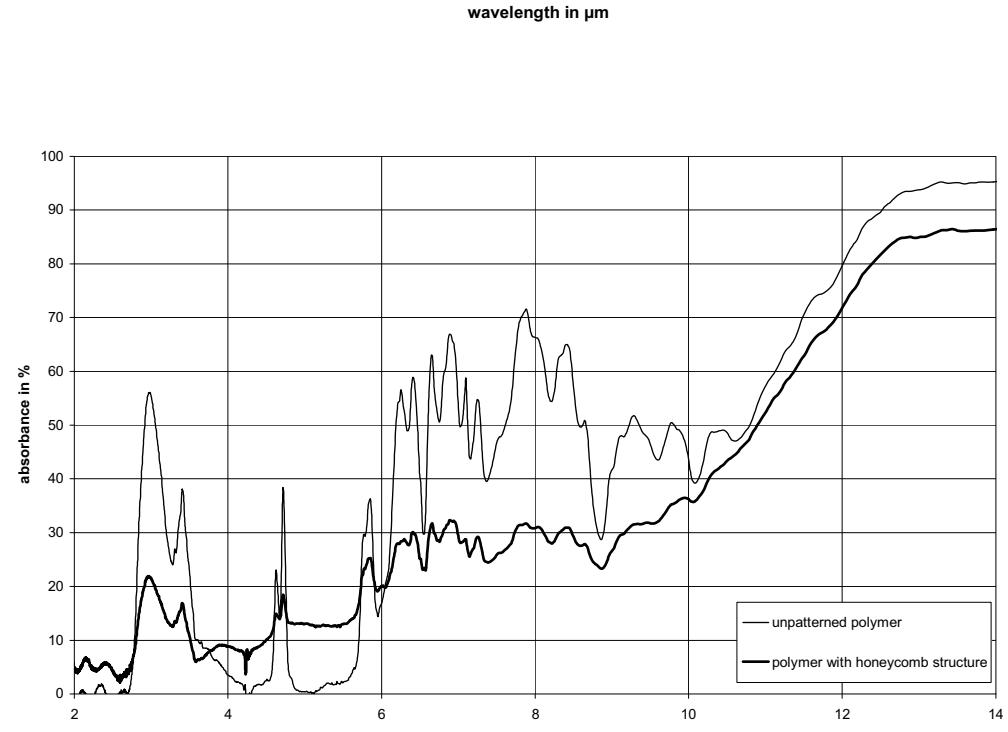

c)

Figure 2: $\quad$ Absorbance of the polymer layer with

a) hole, b) column, and c) honeycomb structures in comparison to an unpatterned layer 


\section{Conclusion}

Patterning of pure polymer layers, which show usually only a mediocre IR absorption, leads to internal reflections and hence to multiple absorptions. In this way, overall absorption can be increased. This principle is known from the structure of moth eyes. Hole, column and honeycomb structures were fabricated by spin-coating and photolithography. Measurements showed that particular hole structures increase the broad-band absorption of novolak polymers up to more than $70 \%$ in the IR range between 2 and $14 \mu \mathrm{m}$.

\section{References}

[1] Walther, L.: Infrarotmesstechnik, Berlin: Verlag Technik, 1983

[2] Plotz, F., u.a.: Thermischer Sensor mit Absorberschicht. Heimann Optoelectronics GmbH, Patent, 1994. 